\title{
Liouville property for solutions of the linearized degenerate thin film equation of fourth order in a halfspace.
}

\author{
S.P.Degtyarev \\ Institute for applied mathematics and mechanics of Ukrainian Na- \\ tional academy of sciences, Donetsk
}

E-mail: degtyar@i.ua

\begin{abstract}
We consider a boundary value problem in the half-space for a linear parabolic equation of fourth order with a degeneration on the boundary of the half-space. The equation under consideration is substantially a linearized thin film equation. We prove that, if the right hand side of the equation and the boundary condition are polynomials in the tangential variables and time, the same property has any solution of a power growth. It is shown also that the specified property does not apply to normal variable. As an application, we present a theorem of uniqueness for the problem in the class of functions of power growth.

The final version is available on Springer at DOI: 10.1007/s00025-015$0467-\mathrm{x}$
\end{abstract}

Key words: Liouville theorem, fourth order, degenerate parabolic equation, thin film equation

MSC: 35B53, 35K35, 35K65, 35Q35

\section{Introduction}

The importance of the classical Liouville theorem is well known. This theorem in complex analysis states that the entire function, growing at infinity no more than a power, is a polynomial. In particular, a bounded entire function is a constant. It is known also that a similar property is inherited by solutions of many linear and nonlinear elliptic and parabolic problems. The presence of such property for solutions of a problem is an extremely important tool for investigation of qualitative properties of such solutions. As a simple example of the use of the partial Liouville theorem of this paper (Theorem 1) we present a result on the uniqueness for the initial-boundary value problems in a halfspace for a fourth order degenerate linear equation in the class of solutions of power growth. The 
literature on the subject is so vast and diverse that we do not try to give a brief overview in this introduction. Among the papers on the Liouville properties of solutions for degenerate equations we mention only the papers [1] - [10] and it is definitely not complete list. We note only that all such papers for degenerate equations are devoted mainly to second-order equations. Author does not know about the Liouville theorems for degenerate parabolic equations of higher than the second order.

In this paper, we consider in the halfspace a boundary value problem for a fourth order parabolic equation with strong degeneration on the boundary of the halfspace. This problem is obtained as a model case under linearization of boundary value problem for the quasilinear degenerate thin film equation in the formulation of the papers [11] - [15]. Denote

$$
R_{+}^{N}=\left\{x=\left(x_{1}, \ldots, x_{N}\right) \in R^{N}: x_{N}>0\right\}, Q=R_{+}^{N} \times R^{1}=\{(x, t): x \in
$$
$\left.R_{+}^{N}, t \in R^{1}\right\}$. For $R>0$ denote also $B_{R}=\left\{x=\left(x^{\prime}, x_{N}\right) \in R_{+}^{N}: \quad 0<x_{N}<R,\left|x^{\prime}\right|<R\right\}$, $x^{\prime}=\left(x_{1}, \ldots, x_{N-1}\right), Q_{R}=\left\{(x, t) \in Q: \quad x \in B_{R},-R^{2}<t<R^{2}\right\}$.

Let a function $u(x, t)$ be defined in $Q, u(x, t) \in W_{\infty, l o c}^{4,1}(Q)$ and let $u(x, t)$ satisfies in this domain to the equation

$$
\frac{\partial u}{\partial t}+\nabla\left(x_{N}^{2} \nabla \Delta u-\beta \nabla u\right)=f(x, t)
$$

where $\nabla=\left(\partial / \partial x_{1}, \ldots, \partial / \partial x_{N}\right), \Delta$ is the Laplace operator in space variables, $\beta \geq 0$ is a given nonnegative constant, $f(x, t)$ is a given function. We suppose that the function $u(x, t)$ possesses the following weighted regularity

$$
\sum_{|\alpha|=4} \max _{Q_{R}}\left|x_{N}^{2} D_{x}^{\alpha} u\right|+\sum_{|\alpha|=3} \max _{Q_{R}}\left|x_{N} D_{x}^{\alpha} u\right| \leq C(R)<\infty
$$

where $\alpha=\left(\alpha_{1}, \ldots, \alpha_{N}\right)$ is a multiindex, $D_{x}^{\alpha} u=\partial^{|\alpha|} u / \partial x_{1}^{\alpha_{1}} \ldots \partial x_{N}^{\alpha_{N}}, C(R)$ is a depending on $R$ constant. We suppose also that the function $u(x, t)$ satisfies on the boundary of the domain $Q$, that is at $x_{N}=0$, to the Dirichlet condition

$$
\left.u(x, t)\right|_{x_{N}=0}=u\left(x^{\prime}, 0, t\right)=g\left(x^{\prime}, t\right),
$$

where $g\left(x^{\prime}, t\right)$ is a given function.

Note here that, as it was shown in the paper [11], for example, in the onedimensional setting, conditions (1.2), (1.3) uniquely determine the solution $u(x, t)$. Thus, the restriction for the class of solution in the form as in (1.2) serves in some sense as a replacement for the second boundary condition, which is necessary for uniformly parabolic fourth order equations of the form (1.1) (in this regard see Remark 2 ). We also note that condition (1.2) can actually be relaxed to, for example, the local weighted integrability conditions for the senior derivatives. It is not our purpose in this paper to find the exact such conditions.

Suppose finally that the function $u(x, t)$ has power growth at infinity 


$$
\max _{Q_{R}}|u(x, t)| \leq C R^{M}, \quad R \geq 1, \quad M>0,
$$

where $C$ is some positive constant, $M$ is a given nonnegative exponent. We agree here that in what follows we denote by the same symbols $C, \nu$, all absolute constants or constants depending only on the fixed initial data of the problem.

Let us formulate now the main result.

Theorem 1 Let the function $f(x, t)$ from (1.1) is a polynomial with respect to the "tangent" variables $x^{\prime}$ and $t$ of degree $M_{f}$, and the function $g\left(x^{\prime}, t\right)$ is a polynomial of degree $M_{g}$. Then, under conditions (1.1) -(1.4), the function $u(x, t)$ is a polynomial with respect to the variables $x^{\prime}$ and $t$ of degree not greater than $M_{u}=$ $[M]$.

Remark 2 Note that the function $u(x, t)$ is not in general a polynomial in the variable $x_{N}$, as it is shown by the following example. Consider a functionv $(x, t) \equiv$ $v\left(x_{N}\right)$, that depends only on the variable $x_{N}$ and satisfies the simplest inhomogeneous equation (1.1), that is

$$
l_{\beta} v \equiv \frac{d}{d x_{N}}\left(x_{N}^{2} \frac{d^{3} v}{d x_{N}^{3}}-\beta \frac{d v}{d x_{N}}\right)=b, \quad b=\text { const } .
$$

The direct calculation shows that for $\beta>0$ the general solution has the form

$$
v\left(x_{N}\right)=C_{1} x_{N}^{a_{1}}+C_{2} x_{N}^{a_{2}}+C_{3} x_{N}+C_{4}-\frac{b}{2 \beta} x_{N}^{2},
$$

where $C_{i}, i=1, \ldots, 4$ are arbitrary constants and the exponents $a_{1}, a_{2}$ are equal to

$$
a_{1}=-\left(\frac{1}{2}+\sqrt{\frac{1}{4}+\beta}\right)<-1, \quad a_{2}=-\frac{1}{2}+\sqrt{\frac{1}{4}+\beta} .
$$

At the same time for $\beta=0$

$$
v\left(x_{N}\right)=C_{1} x_{N} \ln x_{N}+C_{2} x_{N}^{2}+C_{3} x_{N}+C_{4}-\frac{b}{2}\left(x_{N}^{2} \ln x_{N}-\frac{3}{2} x_{N}^{2}\right) .
$$

In this case the conditions on the class of solutions in (1.2) are used to determine one of the arbitrary constants in (1.6), (1.8) (exactly here they are a replacement of the boundary condition). It follows from (1.2) that the constant $C_{1}$ in relations (1.6), (1.8) should be chosen by zero since the corresponding terms do not satisfy (1.2). Thus,

$$
v\left(x_{N}\right)=\left\{\begin{array}{c}
C_{2} x_{N}^{a_{2}}+C_{3} x_{N}+C_{4}-\frac{b}{2 \beta} x_{N}^{2}, \quad \beta>0 \\
C_{2} x_{N}^{2}+C_{3} x_{N}+C_{4}-\frac{b}{2}\left(x_{N}^{2} \ln x_{N}-\frac{3}{2} x_{N}^{2}\right), \quad \beta=0 .
\end{array}\right.
$$


This relation gives an example of a function, satisfying all the conditions of Theorem [1 and which is not a polynomial in the variable $x_{N}$.

As a simple application of Liouville theorem 1 we give a corollary about uniqueness of the solution to the initial-boundary value problems in a halfspace for equation (1.1) in the class of functions with power growth.

\section{Corollary 3 (Uniqueness.)}

Let a function $u(x, t)$ satisfies homogeneous equation (1.1) with $f \equiv 0$ in the halfspace $Q_{+}=Q \cap\{t>0\}$, conditions (1.2), homogeneous condition (1.3) with $g \equiv 0$, and homogeneous initial condition

$$
u(x, 0) \equiv 0, \quad t=0 .
$$

If $u(x, t)$ has at most power growth at infinity (that is, $u(x, t)$ satisfies (1.4)), then this function is identically equal to zero, $u(x, t) \equiv 0$.

\section{Proof.}

Extent the function $u(x, t)$ to the whole domain $Q$, assuming that it is identically zero in $\{t \leq 0\}$, and save its previous designation $u(x, t)$. In view of the homogeneity of equation (1.1) and boundary condition (1.3), it follows from (1.10) that the extended function satisfies homogeneous equation (1.1) with $f \equiv 0$ and homogeneous condition (1.3) with $g \equiv 0$ in the whole domain $Q$. Furthermore, the extended function inherits power growth at infinity. Consequently, such the function $u(x, t)$ satisfies all the conditions of Theorem 1 and therefore is a polynomial in the variable $t$ for all values of the variables $x$. Since this polynomial is identically equal to zero in $\{t \leq 0\}$, we see that it is identically equal to zero for all $t$. Thus, the function $u(x, t)$ is identically equal to zero.

To further content of the article is constructed as follows. In the second section we present the proof of Theorem 1. This proof is based on local integral estimates for derivatives of the solution $u(x, t)$ with respect to the "tangent" variables $x^{\prime}=\left(x_{1}, \ldots, x_{N-1}\right)$ and with respect to the variable $t$ via a local integral norm of the solution itself. The proof of the necessary local integral estimates is presented for convenience in the third final section.

\section{The proof of Theorem 1 .}

Denote for $R>0$, as before, $B_{R}=\left\{x=\left(x^{\prime}, x_{N}\right) \in R_{+}^{N}: \quad 0<x_{N}<R,\left|x^{\prime}\right|<R\right\}$, $x^{\prime}=\left(x_{1}, \ldots, x_{N-1}\right), Q_{R}=\left\{(x, t) \in Q: \quad x \in B_{R},-R^{2}<t<R^{2}\right\}$. The proof of Theorem 1 is based on the following lemma.

Lemma 4 Let the function $u(x, t)$ satisfies the conditions of Theorem 1 except for power growth at infinity. Let, besides, function $u(x, t)$ be infinitely differentiable 
in the variables $x^{\prime}, t$ in $\bar{Q}$ and its derivatives with respect to these variables belong to the same space as $u(x, t)$ itself. Suppose, in addition, $f \equiv 0$ in equation (1.1) and $g \equiv 0$ in condition (1.3).

Then for all $R>1, q>1$ the following estimates are valid

$$
\int_{Q_{R}}|\nabla u|^{2} d x d t \leq \frac{C_{q}}{R^{2}} \int_{Q_{q R}} u^{2} d x d t, \quad \int_{Q_{R}} u_{t}^{2} d x d t \leq \frac{C_{q}}{R^{4}} \int_{Q_{q R}} u^{2} d x d t .
$$

The proof of this lemma will be given in the next section, but now proceed to the proof of Theorem 1 .

Suppose first that the function $u(x, t)$ is infinitely differentiable with respect to the variables $x^{\prime}, t$ in $\bar{Q}$ and its derivatives with respect to these variables belong to the same space that $u(x, t)$ itself. And let also $f \equiv 0$ in equation (1.1) and $g \equiv 0$ in condition (1.3), that is, $u(x, t)$ satisfies the conditions of Lemma 4 . As the coefficients of equation (1.1) does not depend on $x^{\prime}, t$, differentiating this equation with respect to these variables and using estimates (2.1) iteratively, we obtain by induction

$$
\int_{Q_{R}}\left|D_{x^{\prime}}^{\alpha} D_{t}^{\beta} u\right|^{2} d x d t \leq \frac{C(q, \alpha, \beta)}{R^{2|\alpha|+4 \beta}} \int_{Q_{q^{|\alpha|+\beta} R}} u^{2} d x d t
$$

where $\alpha=\left(\alpha_{1}, \ldots, \alpha_{N-1}\right)$ is a multiindex, $D_{x^{\prime}}^{\alpha}=D_{x_{1}}^{\alpha_{1} \ldots} D_{x_{N-1}}^{\alpha_{N-1}}$. At the same time, condition (1.4) implies the estimate of the integral on the right side of (2.2)

$$
\int_{Q_{q}|\alpha|+\beta_{R}} u^{2} d x d t \leq C(q, \alpha, \beta) R^{2 M+N+2} .
$$

And then we obtain from (2.2)

$$
\int_{Q_{R}}\left|D_{x^{\prime}}^{\alpha} D_{t}^{\beta} u\right|^{2} d x d t \leq C(q, \alpha, \beta) R^{2 M+N+2-(2|\alpha|+4 \beta)} .
$$

Choose now in the last estimate sufficiently big $|\alpha|$ and $\beta$. Letting $R$ to infinity, we obtain $D_{x^{\prime}}^{\alpha} D_{t}^{\beta} u \equiv 0$ in $Q$ for $2|\alpha|+4 \beta>2 M+N+2$. This means that the function $u(x, t)$ is a polynomial in the variables $x^{\prime}, t$ and the degree of this polynomial $M_{u} \leq[M]$ by the estimate (1.4). Thus, Theorem 1 is proved under our additional assumptions. Let us remove now our additional assumptions.

Let $u(x, t)$ satisfies the conditions of Theorem 1 and let $\omega\left(x^{\prime}, t\right)$ be a function of the class $C_{0}^{\infty}\left(R^{N-1} \times R^{1}\right)$ with the compact support in the set $\left\{\left(x^{\prime}, t\right):\left|x^{\prime}\right|+|t|<1\right\}$ and with the properties (mollifier)

$$
\int_{R^{N-1} \times R^{1}} \omega\left(x^{\prime}, t\right) d x^{\prime} d t=\int_{\left|x^{\prime}\right|+|t|<1} \omega\left(x^{\prime}, t\right) d x^{\prime} d t=1 .
$$


For $\varepsilon \in(0,1)$ denote $\omega_{\varepsilon}\left(x^{\prime}, t\right)=\varepsilon^{-N} \omega\left(x^{\prime} / \varepsilon, t / \varepsilon\right)$ and consider the function (th smoothing with respect to "tangent" variables)

$$
u_{\varepsilon}(x, t)=\omega_{\varepsilon} * u=\int_{R^{N-1} \times R^{1}} \omega_{\varepsilon}\left(x^{\prime}-\xi^{\prime}, t-\tau\right) u\left(\xi^{\prime}, x_{N}, \tau\right) d \xi d \tau .
$$

It follows from well-known properties of convolution that the function $u_{\varepsilon}(x, t)$ has the following properties.

1. The function $u_{\varepsilon}(x, t)$ is infinitely differentiable in the variables $x^{\prime}$ and $t$, its derivatives in these variables belong to the same class as the $u(x, t)$ and satisfy (1.2) with a constant, independent of $\varepsilon \in(0,1)$.

2. Since the coefficients of equation (1.1) for $u(x, t)$ do not depend on $x^{\prime}$ and $t$, the function $u_{\varepsilon}(x, t)$ satisfies equation (1.1) with the right hand side $f_{\varepsilon}(x, t)$ and boundary condition (1.3) with the function $g_{\varepsilon}\left(x^{\prime}, t\right)$, where

$$
\begin{array}{r}
f_{\varepsilon}(x, t)=\int_{R^{N-1} \times R^{1}} \omega_{\varepsilon}\left(\xi^{\prime}, \tau\right) f\left(x^{\prime}-\xi^{\prime}, x_{N}, t-\tau\right) d \xi d \tau, \\
g_{\varepsilon}\left(x^{\prime}, t\right)=\int_{R^{N-1} \times R^{1}} \omega_{\varepsilon}\left(\xi^{\prime}, \tau\right) g\left(x^{\prime}-\xi^{\prime}, t-\tau\right) d \xi d \tau .
\end{array}
$$

At the same time it follows from (2.3) that the functions $f_{\varepsilon}(x, t)$ and $g_{\varepsilon}\left(x^{\prime}, t\right)$ are polynomials in the variables $x^{\prime}, t$ and the degrees of these polynomials coincide with those of $f(x, t)$ and $g\left(x^{\prime}, t\right)$. So the degrees of these polynomials do not depend on $\varepsilon \in(0,1)$.

3. It follows from the properties of the function $\omega_{\varepsilon}\left(x^{\prime}, t\right)$ and from the definition of the function $u_{\varepsilon}(x, t)$ that $u_{\varepsilon}(x, t)$ satisfies condition (1.4) with a constant $C$ and the exponent $M$, which do not depend on $\varepsilon \in(0,1)$. Moreover,

$$
D_{x^{\prime}}^{\alpha} D_{t}^{\beta} u_{\varepsilon}(x, t)=\int_{R^{N-1} \times R^{1}} D_{x^{\prime}}^{\alpha} D_{t}^{\beta} \omega_{\varepsilon}\left(x^{\prime}-\xi^{\prime}, t-\tau\right) u\left(\xi^{\prime}, x_{N}, \tau\right) d \xi d \tau
$$

and so

$$
\max _{Q_{R}}\left|D_{x^{\prime}}^{\alpha} D_{t}^{\beta} u_{\varepsilon}(x, t)\right| \leq \frac{C}{\varepsilon^{|\alpha|+\beta}} R^{M}
$$

Suppose now that the multiindex $\alpha_{0}$ and $\beta_{0}$ are chosen so large comparatively with the degrees $M_{f}$ and $M_{g}$ of polynomials $f_{\varepsilon}(x, t)$ and $g_{\varepsilon}\left(x^{\prime}, t\right)$ that $D_{x^{\prime}}^{\alpha_{0}} D_{t}^{\beta_{0}} f_{\varepsilon}(x, t) \equiv 0, D_{x^{\prime}}^{\alpha_{0}} D_{t}^{\beta_{0}} g_{\varepsilon}\left(x^{\prime}, t\right) \equiv 0$. Denote $v_{\varepsilon}(x, t)=D_{x^{\prime}}^{\alpha_{0}} D_{t}^{\beta_{0}} u_{\varepsilon}(x, t)$. It follows from the above mentioned properties of the function $u_{\varepsilon}(x, t)$ that $v_{\varepsilon}(x, t)$ has all the same properties that the function $u_{\varepsilon}(x, t)$, including (2.4). The difference is that $v_{\varepsilon}(x, t)$ satisfies equation (1.1) with zero right hand side and it satisfies zero boundary condition (1.3). Thus, by the above, the function $v_{\varepsilon}(x, t)$ 
is a polynomial in the variables $x^{\prime}$ and $t$. But then, by the definition of $v_{\varepsilon}(x, t)$, the function $u_{\varepsilon}(x, t)$ is also a polynomial in these variables, and its degree does not depend on $\varepsilon \in(0,1)$ and does not exceed $[M]$, by virtue of (2.4).

Let $\varphi(x, t)$ be an arbitrary function from $C_{0}^{\infty}(Q)$ and let $m=[M]+1$. Since $D_{x_{1}}^{m} \ldots D_{x_{N-1}}^{m} D_{t}^{m} u_{\varepsilon}(x, t) \equiv 0$, multiplying this identity by $\varphi(x, t)$ and integrating by parts over the domain $Q$, we obtain

$$
\int_{Q} u_{\varepsilon}(x, t) D_{x_{1}}^{m} \ldots D_{x_{N-1}}^{m} D_{t}^{m} \varphi(x, t) d x d t=0 .
$$

Since $u(x, t) \in L_{2, l o c}(Q)$, the averaged functions $u_{\varepsilon}(x, t)$ tend in this space to the original function $u(x, t)$ as $\varepsilon \rightarrow 0$, as it is well known. Therefore, taking in the last inequality the limit as $\varepsilon \rightarrow 0$, we get

$$
\int_{Q} u(x, t) D_{x_{1}}^{m} \ldots D_{x_{N-1}}^{m} D_{t}^{m} \varphi(x, t) d x d t=0 .
$$

As the function $\varphi(x, t)$ is arbitrary, we infer that $D_{x_{1}}^{m} \ldots D_{x_{N-1}}^{m} D_{t}^{m} u(x, t) \equiv 0$ in the sense of distributions. But it is well known that this means that the function $u(x, t)$ is a polynomial with respect to the variables $x^{\prime}$ and $t$. Moreover, in view of (1.4) the degree of this polynomial does not exceed $[M]$.

Thus Theorem 1 is proved under the assumption of validity of Lemma 4 .

\section{Proof of Lemma 4.}

In this section, we prove Lemma 4 and this will complete the proof of Theorem 1 .

We need in the future some corollary of the following statement ([16], Lemma $3.1)$.

Lemma 5 Let $f(t)$ be a nonnegative bounded function with the domain $\left[r_{0}, r_{1}\right]$, $r_{0} \geq 0$. Suppose that for $r_{0} \leq t<s \leq r_{1}$

$$
f(t) \leq \theta f(s)+\left[A(s-t)^{-a}+B\right],
$$

where $A, B, a, \theta$ are some nonnegative constants and $0 \leq \theta<1$. Then for all $r_{0} \leq t<s \leq r_{1}$

$$
f(t) \leq C_{\theta, a}\left[A(s-t)^{-a}+B\right]
$$

We will use this lemma in the following particular case. Recall that for $R>0$ we denote $B_{R}=\left\{x=\left(x^{\prime}, x_{N}\right) \in R_{+}^{N}: \quad 0<x_{N}<R,\left|x^{\prime}\right|<R\right\}, x^{\prime}=$ $\left(x_{1}, \ldots, x_{N-1}\right), Q_{R}=\left\{(x, t) \in Q: \quad x \in B_{R},-R^{2}<t<R^{2}\right\}$. 
Lemma 6 Let functions $u(x, t) \geq 0, U(x, t) \geq 0$ are locally integrable in $Q$ and for all $R>0, q \in(1,3)$ we have the estimate

$$
\int_{Q_{R}} u(x, t) d x d t \leq \varepsilon \int_{Q_{q R}} u(x, t) d x d t+\frac{C}{(q-1)^{a} R^{b}} \int_{Q_{q R}} U(x, t) d x d t+B,
$$

where $\varepsilon \in(0,1), B \geq 0$.

Then for all $R>0, q \in(1,3)$

$$
\int_{Q_{R}} u(x, t) d x d t \leq \frac{C_{a, \varepsilon}}{(q-1)^{a} R^{b}} \int_{Q_{q R}} U(x, t) d x d t+B .
$$

This lemma is immediate consequence of Lemma 5. Keeping in mind that $R$ and $q$ in (3.1) are arbitrary, it is enough to consider on the interval $[1, q]$ the function $f(t)=\int_{Q_{t R}} u(x, \tau) d x d \tau$ and take into account that for $t \in[1, q]$ we have $\int_{Q_{t R}} U(x, \tau) d x d \tau \leq A=\int_{Q_{q R}} U(x, t) d x d t$.

Note that assertions analogous to Lemma 6 was implicitly used before in the papers [17]- 25], for example,.

We will use also the well known Nirenberg-Gagliardo inequality in it's particular case (see, for example,[26] or [27], Theorem 5.2)

$$
\int_{B_{R}}|\nabla v|^{2} d x \leq C\left(\int_{B_{R}}\left|D_{x}^{2} v\right|^{2} d x\right)^{\frac{1}{2}}\left(\int_{B_{R}} v^{2} d x\right)^{\frac{1}{2}}
$$

where $\left|D_{x}^{2} v\right|^{2} \equiv \sum_{|\alpha|=2}\left|D_{x}^{\alpha} v\right|^{2}$ and the constant $C$ does not depend on $R$. This inequality is valid for functions $v(x, t)$, that vanish on the boundary $\partial B_{R}$ together with their first derivatives. It is well known from the theory of Dirichlet problem for the Poisson equation that for such functions

$$
\int_{B_{R}}\left|D_{x}^{2} v\right|^{2} d x \leq C \int_{B_{R}}(\Delta v)^{2} d x
$$

where the constant $C$ again does not depend on $R$. Substituting (3.4) in (3.3) and using the Cauchy inequality with $\varepsilon$ to estimate the product on the right hand side of (3.3), we obtain

$$
\int_{B_{R}}|\nabla v|^{2} d x \leq \varepsilon \int_{B_{R}}(\Delta v)^{2} d x+\frac{-}{\varepsilon} \int_{B_{R}} v^{2} d x .
$$

Integrating this inequality in $t$ from $-R^{2}$ t5o $R^{2}$, we arrive at the inequality 


$$
\int_{Q_{R}}|\nabla v|^{2} d x d t \leq \varepsilon \int_{Q_{R}}(\Delta v)^{2} d x d t+\frac{C}{\varepsilon} \int_{Q_{R}} v^{2} d x d t, \quad \varepsilon>0 .
$$

We will use also the well known Hardy inequality in the form (see, for example, [26] or [28], formula $(0.3)$ )

$$
\int_{0}^{R} w^{2} d x_{N} \leq 4 \int_{0}^{R} x_{N}^{2}\left(\frac{\partial w}{\partial x_{N}}\right)^{2} d x
$$

This inequality is valid for such functions $w(x, t)$ that $\left.w\right|_{x_{N}=R}=0$. Integrating this inequality in $x^{\prime} \in\left\{\left|x^{\prime}\right|<R\right\}$ and in $t$ from $-R^{2}$ to $R^{2}$, we obtain

$$
\int_{Q_{R}} w^{2} d x d t \leq 4 \int_{Q_{R}} x_{N}^{2}\left(\frac{\partial w}{\partial x_{N}}\right)^{2} d x d t
$$

Turning to the proof of Lemma 4, let us agree to denote everywhere below for brevity $C_{q}=C /(q-1)^{a}$, where $a$ is a nonnegative number.

The proof of Lemma 4 will be obtained as a result of a collection of local integral estimates with the using of inequalities (3.5), (3.6) an equation (1.1) together with the boundary condition (1.3). In what follows $u(x, t)$ is some fixed function and it satisfies the conditions of Lemma 4 .

Let $q \in(1,3), R>1$, and let $s>0$ be sufficiently large. Let also $\eta(x, t)$ be such a nonnegative function of the class $C^{\infty}(\bar{Q})$ that

$$
\begin{gathered}
0 \leq \eta \leq 1,\left.\quad \eta(x, t)\right|_{Q_{R}} \equiv 1,\left.\quad \eta(x, t)\right|_{Q \backslash Q_{q R}} \equiv 0, \\
\left|D_{x}^{\alpha} D_{t}^{\beta} \eta\right| \leq \frac{C_{\alpha, \beta}}{[(q-1) R]^{|\alpha|+2 \beta}} \equiv \frac{C_{q}}{R^{|\alpha|+2 \beta}} .
\end{gathered}
$$

Let us agree for brevity to call such a function cut-off function for the cylinder $Q_{R}$. Consider the function $v(x, t)=u(x, t) \eta^{s}(x, t)$. Applying to this function inequality (3.5), we obtain after simple calculations with the using (3.7)

$$
\int_{Q_{R}}|\nabla u|^{2} d x d t \leq \varepsilon \int_{Q_{q R}}(\Delta u)^{2} d x d t+\varepsilon \frac{C_{q}}{R^{2}} \int_{Q_{q R}}|\nabla u|^{2} d x d t+\left(\bar{\varepsilon}+\frac{C_{q}}{R^{4}}\right) \int_{Q_{q R}} u^{2} d x d t .
$$

Choosing in this estimate $\varepsilon=\varepsilon_{1} R^{2} / C_{q}, \varepsilon_{1} \in(0,1)$ and taking into account that $R>1$, we arrive at the inequality

$$
\int_{Q_{R}}|\nabla u|^{2} d x d t \leq \varepsilon_{1} R^{2} C \int_{Q_{q R}}(\Delta u)^{2} d x d t+\varepsilon_{1} \int_{Q_{q R}}|\nabla u|^{2} d x d t+\frac{C_{q}}{\varepsilon_{1} R^{2}} \int_{Q_{q R}} u^{2} d x d t .
$$


Now it follows from the last inequality and Lemma 6 that

$$
\int_{Q_{R}}|\nabla u|^{2} d x d t \leq \varepsilon_{1} R^{2} C \int_{Q_{q R}}(\Delta u)^{2} d x d t+\frac{C_{q}}{\varepsilon_{1} R^{2}} \int_{Q_{q R}} u^{2} d x d t .
$$

Let now $\eta(x, t)$ be a cut-off function of the cylinder $Q_{q^{2} R}$ and it identically equal to 1 on $Q_{q R}$. Denote $w(x, t)=\eta^{s}(x, t) \Delta u$ and apply to this function inequality (3.6) in the domain $Q_{q^{2} R}$. After simple calculations with the using (3.7), we obtain

$$
\int_{Q_{q R}}(\Delta u)^{2} d x d t \leq \int_{Q_{q^{2} R}} x_{N}^{2}\left(\frac{\partial}{\partial x_{N}} \Delta u\right)^{2} d x d t+\frac{C}{R^{2}} \int_{Q_{q^{2} R}} x_{N}^{2}(\Delta u)^{2} d x d t .
$$

As $q \in(1,3)$ is arbitrary, substituting this estimate in (3.8) and denoting $q^{2}$ again by $q$, we get the inequality

$$
\begin{gathered}
\int_{Q_{R}}|\nabla u|^{2} d x d t \leq \varepsilon_{1} R^{2} C \int_{Q_{q R}} x_{N}^{2}|\nabla \Delta u|^{2} d x d t+\varepsilon_{1} C \int_{Q_{q R}} x_{N}^{2}(\Delta u)^{2} d x d t+ \\
+\frac{C_{q}}{\varepsilon_{1} R^{2}} \int_{Q_{q R}} u^{2} d x d t .
\end{gathered}
$$

This is the first from several integral inequalities we need. It is obtained from Sobolev embeddings and we did not apply equation (1.1).

Now our goal is to use the equation to estimate the first and the second terms on the right hand side of (3.9) and also to obtain an analogous estimate for the time derivative. At this we will use also boundary condition (1.3) and the fact that it follows from (1.2) that

$$
\left.x_{N}^{2} D_{x}^{\alpha} u\right|_{x_{N}=0}=0, \quad|\alpha| \leq 3 ;\left.\quad x_{N} D_{x}^{\alpha} u\right|_{x_{N}=0}=0, \quad|\alpha| \leq 2 .
$$

Recall besides that according to the conditions of the lemma the function $u$ is infinitely differentiable with respect to the "tangent" variables $x^{\prime}$ and $t$ in combinations with derivatives with respect to $x_{N}$ up to the fourth order.

Let $\eta(x, t)$ be defined in (3.7). Multiply equation (1.1) by the function $u(x, t) \eta^{s}(x, t)$ and integrate by parts. Taking into account (1.3), (3.10) and the fact that the support of $\eta(x, t)$ is a compact set, we obtain

$$
-s \int_{Q_{q R}} u^{2} \eta_{t} \eta^{s-1} d x d t-\int_{Q_{q R}} x_{N}^{2} \nabla \Delta u \nabla u \eta^{s} d x d t-s \int_{Q_{q R}} x_{N}^{2} \nabla \Delta u \nabla \eta u \eta^{s-1} d x d t+
$$




$$
+\beta \int_{Q_{q R}}|\nabla u|^{2} \eta^{s} d x d t+\beta s \int_{Q_{q R}} \nabla u \nabla \eta u \eta^{s-1} d x d t=0 .
$$

Integrating once more by parts in the terms with the expression $\nabla \Delta u$ and taking all terms without the expressions $(\Delta u)^{2} \eta^{s}$ and $\beta|\nabla u|^{2} \eta^{s}$ to the right hand side, we get

$$
\begin{gathered}
\int_{Q_{q R}} x_{N}^{2}(\Delta u)^{2} \eta^{s} d x d t+\beta \int_{Q_{q R}}|\nabla u|^{2} \eta^{s} d x d t=-2 \int_{Q_{q R}} x_{N} \Delta u \frac{\partial u}{\partial x_{N}} \eta^{s} d x d t- \\
-s \int_{Q_{q R}} x_{N}^{2} \Delta u\left[\Delta \eta \eta^{s-1}+(s-1)(\nabla \eta)^{2} \eta^{s-2}\right] u d x d t-2 s \int_{Q_{q R}} x_{N}^{2} \Delta u \nabla u \nabla \eta \eta^{s-1} d x d t- \\
-2 s \int_{Q_{q R}} x_{N} \Delta u \frac{\partial \eta}{\partial x_{N}} u \eta^{s-1} d x d t+s \int_{Q_{q R}} u^{2} \eta_{t} \eta^{s-1} d x d t- \\
-\beta s \int_{Q_{q R}} \nabla u \nabla \eta u \eta^{s-1} d x d t \equiv I_{1}+I_{2}+I_{3}+I_{4}+I_{5}+I_{6} .
\end{gathered}
$$

We estimate the integrals $I_{1}-I_{6}$ on the right hand side of (3.11) with the help of the integral Hölder inequality with the exponents $p=p^{\prime}=2$ and with the help of Cauchy's inequality with $\varepsilon$, taking into account properties (3.7) of the function $\eta(x, t)$. For example, we have for $I_{4}$

$$
\begin{gathered}
\left|I_{4}\right| \leq \int_{Q_{q R}}\left(\left|x_{N} \Delta u\right| \eta^{s / 2}\right)\left(u\left|\frac{\partial \eta}{\partial x_{N}}\right| \eta^{s / 2-1}\right) d x d t \leq \\
\leq C\left(\int_{Q_{q R}} x_{N}^{2}(\Delta u)^{2} \eta^{s} d x d t\right)^{\frac{1}{2}}\left(\int_{Q_{q R}} u^{2}\left|\frac{\partial \eta}{\partial x_{N}}\right|^{2} \eta^{s-2} d x d t\right)^{\frac{1}{2}} \leq \\
\leq C\left(\int_{Q_{q R}} x_{N}^{2}(\Delta u)^{2} \eta^{s} d x d t\right)^{\frac{1}{2}}\left(\frac{C_{q}}{R^{2}} \int_{Q_{q R}} u^{2} d x d t\right)^{\frac{1}{2}} \leq \\
\leq \int_{Q_{q R}} x_{N}^{2}(\Delta u)^{2} \eta^{s} d x d t+\frac{C_{q}}{\varepsilon R^{2}} \int_{Q_{q R}} u^{2} d x d t .
\end{gathered}
$$


Completely analogous estimates for the other integrals together with the fact that $x_{N} \leq C R$ on $Q_{q R}$ and with (3.11) give

$$
\begin{gathered}
\int_{Q_{q R}} x_{N}^{2}(\Delta u)^{2} \eta^{s} d x d t+\beta \int_{Q_{q R}}|\nabla u|^{2} \eta^{s} d x d t \leq \\
\leq \varepsilon \int_{Q_{q R}} x_{N}^{2}(\Delta u)^{2} \eta^{s} d x d t+\frac{C_{q}}{\varepsilon} \int_{Q_{q R}}|\nabla u|^{2} d x d t+\frac{C_{q}}{\varepsilon R^{2}} \int_{Q_{q R}} u^{2} d x d t .
\end{gathered}
$$

Choose in this estimate $\varepsilon$ such that $\varepsilon=1 / 2$ and move the first term on the right hand side to the left. Then, taking into account properties of $\eta$, we finally obtain

$$
\int_{Q_{R}} x_{N}^{2}(\Delta u)^{2} d x d t+\beta \int_{Q_{R}}|\nabla u|^{2} d x d t \leq C_{q} \int_{Q_{q R}}|\nabla u|^{2} d x d t+\frac{C_{q}}{R^{2}} \int_{Q_{q R}} u^{2} d x d t .
$$

Turn now to the following estimate. Using the same function $\eta$ as before, multiply equation (1.1) by $(\Delta u) \eta^{s}$ integrate over $Q_{R}$ and integrate by parts in the variables $x$ in terms without $\beta$. Bearing in mind that $\left.u_{t}\right|_{x_{N}=0}=0$ in view of boundary condition (1.3), we have

$$
\begin{gathered}
-\int_{Q_{q R}} \nabla u_{t} \nabla u \eta^{s} d x d t-s \int_{Q_{q R}} u_{t} \nabla u \nabla \eta \eta^{s-1} d x d t-\int_{Q_{q R}} x_{N}^{2}(\nabla \Delta u)^{2} \eta^{s} d x d t- \\
-s \int_{Q_{q R}} x_{N}^{2} \nabla \Delta u \nabla \eta \Delta u \eta^{s-1} d x d t-\beta \int_{Q_{q R}}(\Delta u)^{2} \eta^{s} d x d t=0 .
\end{gathered}
$$

Substituting in the first term $\nabla u_{t} \nabla u=\left[(\nabla u)^{2} / 2\right]_{t}$ and integrating by parts in $t$ in this term, we can represent the last equality as

$$
\begin{gathered}
\int_{Q_{q R}} x_{N}^{2}(\nabla \Delta u)^{2} \eta^{s} d x d t+\beta \int_{Q_{q R}}(\Delta u)^{2} \eta^{s} d x d t=-s \int_{Q_{q R}} u_{t} \nabla u \nabla \eta \eta^{s-1} d x d t- \\
-s \int_{Q_{q R}} x_{N}^{2} \nabla \Delta u \nabla \eta \Delta u \eta^{s-1} d x d t+\frac{s}{2} \int_{Q_{q R}}(\nabla u)^{2} \eta_{t} \eta^{s-1} d x d t \equiv I_{1}+I_{2}+I_{3} .
\end{gathered}
$$

The integrals $I_{1}-I_{3}$ are estimated as before. In particular,

$$
\left|I_{1}\right| \leq C_{q} \int_{Q_{q R}}\left|u_{t}\right| \frac{|\nabla u|}{R} d x d t \leq \varepsilon_{2} \int_{Q_{q R}} u_{t}^{2} d x d t+\frac{C_{q}}{\varepsilon_{2} R^{2}} \int_{Q_{q R}}(\nabla u)^{2} d x d t,
$$




$$
\begin{gathered}
\left|I_{2}\right| \leq \varepsilon \int_{Q_{q R}} x_{N}^{2}(\nabla \Delta u)^{2} \eta^{s} d x d t+\frac{C_{q}}{\varepsilon R^{2}} \int_{Q_{q R}} x_{N}^{2}(\Delta u)^{2} d x d t \\
\left|I_{3}\right| \leq \frac{C_{q}}{R^{2}} \int_{Q_{q R}}(\nabla u)^{2} d x d t
\end{gathered}
$$

Thus, we get from (3.14)

$$
\begin{gathered}
\int_{Q_{q R}} x_{N}^{2}(\nabla \Delta u)^{2} \eta^{s} d x d t+\beta \int_{Q_{q R}}(\Delta u)^{2} \eta^{s} d x d t \leq \varepsilon \int_{Q_{q R}} x_{N}^{2}(\nabla \Delta u)^{2} \eta^{s} d x d t+ \\
+\frac{C_{q}}{\varepsilon R^{2}} \int_{Q_{q R}} x_{N}^{2}(\Delta u)^{2} d x d t+\varepsilon_{2} \int_{Q_{q R}} u_{t}^{2} d x d t+\frac{C_{q}}{\varepsilon_{2} R^{2}} \int_{Q_{q R}}(\nabla u)^{2} d x d t .
\end{gathered}
$$

The first term on the right can be moved to the left side with the choice $\varepsilon=1 / 2$. As for the second term on the right, we use (3.13) with $q R$ instead of $R$ to estimate it. This gives

$$
\begin{aligned}
\int_{Q_{q R}} x_{N}^{2}(\nabla \Delta u)^{2} \eta^{s} d x d t & +\beta \int_{Q_{q R}}(\Delta u)^{2} \eta^{s} d x d t \leq \varepsilon_{2} \int_{Q_{q R}} u_{t}^{2} d x d t+\frac{C_{q}}{\varepsilon_{2} R^{2}} \int_{Q_{q R}}(\nabla u)^{2} d x d t+ \\
& +\frac{C_{q}}{R^{2}} \int_{Q_{q^{2} R}}|\nabla u|^{2} d x d t+\frac{C_{q}}{R^{4}} \int_{Q_{q^{2} R}} u^{2} d x d t .
\end{aligned}
$$

Now we the properties of the function $\eta\left(\eta \equiv 1\right.$ on $\left.Q_{R}\right)$, then we estimate the integrals over $Q_{q R}$ on the right hand side by the same integrals over $Q_{q^{2} R}$ and as $q$ is arbitrary, we denote $q^{2}$ again by $q$. We obtain finally

$$
\begin{gathered}
\int_{Q_{R}} x_{N}^{2}(\nabla \Delta u)^{2} d x d t+\beta \int_{Q_{R}}(\Delta u)^{2} d x d t \leq \\
\leq \varepsilon_{2} \int_{Q_{q R}} u_{t}^{2} d x d t+\frac{C_{q}}{\varepsilon_{2} R^{2}} \int_{Q_{q R}}(\nabla u)^{2} d x d t+\frac{C_{q}}{R^{4}} \int_{Q_{q R}} u^{2} d x d t .
\end{gathered}
$$

We now turn to the following estimate. Recall that the function $u$ is infinitely differentiable in the variables $\left(x^{\prime}, t\right)$ and it satisfies the boundary conditions $\left.u\right|_{x_{N}=0}=\left.u_{t}\right|_{x_{N}=0}=0$. Let $\eta$ be the same function as above. Multiply equation (1.1) by $\Delta u_{t} \eta^{s}$ and integrate by parts with respect to the space variables in the first and in the second terms. We obtain 


$$
\begin{gathered}
\int_{Q_{q R}}\left(\nabla u_{t}\right)^{2} \eta^{s} d x d t+\int_{Q_{q R}} x_{N}^{2}\left(\nabla \Delta u, \nabla \Delta u_{t}\right) \eta^{s} d x d t+\beta \int_{Q_{q R}} \Delta u \Delta u_{t} \eta^{s} d x d t= \\
=-s \int_{Q_{q R}} \nabla u_{t} \nabla \eta u_{t} \eta^{s-1} d x d t-s \int_{Q_{q R}} x_{N}^{2}(\nabla \Delta u, \nabla \eta) \Delta u_{t} \eta^{s-1} d x d t .
\end{gathered}
$$

Integrating now by parts with respect to $t$ in the second and in the third terms on the left and moving the results to the right hand side, we obtain

$$
\begin{aligned}
& \int_{Q_{q R}}\left(\nabla u_{t}\right)^{2} \eta^{s} d x d t=-s \int_{Q_{q R}} \nabla u_{t} \nabla \eta u_{t} \eta^{s-1} d x d t-s \int_{Q_{q R}} x_{N}^{2}(\nabla \Delta u, \nabla \eta) \Delta u_{t} \eta^{s-1} d x d t+ \\
& \quad+\frac{1}{2} \int_{Q_{q R}} x_{N}^{2}(\nabla \Delta u)^{2} \eta_{t} \eta^{s-1} d x d t+\frac{\beta}{2} \int_{Q_{q R}}(\Delta u)^{2} \eta_{t} \eta^{s-1} d x d t \equiv I_{1}+I_{2}+I_{3}+I_{4} \cdot \text { (3.16) }
\end{aligned}
$$

The integrals $I_{1}-I_{4}$ are estimated in the same way as before. This gives

$$
\begin{gathered}
\left|I_{1}\right| \leq \frac{1}{2} \int_{Q_{q R}}\left(\nabla u_{t}\right)^{2} \eta^{s} d x d t+\frac{C_{q}}{R^{2}} \int_{Q_{q R}}\left(u_{t}\right)^{2} d x d t \\
\left|I_{2}\right| \leq \varepsilon_{3} \int_{Q_{q R}} x_{N}^{2}\left(\Delta u_{t}\right)^{2} d x d t+\frac{C_{q}}{\varepsilon_{3} R^{2}} \int_{Q_{q R}} x_{N}^{2}(\nabla \Delta u)^{2} d x d t \\
\left|I_{3}\right| \leq \frac{C_{q}}{R^{2}} \int_{Q_{q R}} x_{N}^{2}(\nabla \Delta u)^{2} d x d t \\
\left|I_{4}\right| \leq \frac{C_{q}}{R^{2}} \int_{Q_{q R}}(\Delta u)^{2} d x d t
\end{gathered}
$$

Consider the integral $I_{4}$. Let $\eta_{q}$ be a function, which is analogous to $\eta$ with the replacing $R$ with $q R$. In particular, $\eta_{q} \equiv 1$ on $Q_{q R}$ and $\eta_{q} \equiv 0$ outside $Q_{q^{2} R}$. Using (3.6) and (3.7) for $\eta_{q}$, we proceed with the estimate for $I_{4}$ in the following way

$$
\left|I_{4}\right| \leq \frac{C_{q}}{R^{2}} \int_{Q_{q R}}\left(\Delta u \eta_{q}^{s}\right)^{2} d x d t \leq \frac{C_{q}}{R^{2}} \int_{Q_{q^{2} R}}\left(\Delta u \eta_{q}^{s}\right)^{2} d x d t \leq
$$




$$
\begin{gathered}
\leq \frac{C_{q}}{R^{2}} \int_{Q_{q^{2} R}} x_{N}^{2}\left(\nabla\left(\Delta u \eta_{q}^{s}\right)\right)^{2} d x d t \leq \\
\leq \frac{C_{q}}{R^{2}} \int_{Q_{q^{2} R}} x_{N}^{2}(\nabla \Delta u)^{2} d x d t+\frac{C_{q}}{R^{4}} \int_{Q_{q^{2} R}} x_{N}^{2}(\Delta u)^{2} d x d t .
\end{gathered}
$$

We estimate now the right hand side of (3.16) with the help of the estimates for the integrals $I_{1}-I_{4}$. In this way we move the first integral in (3.17) to the right hand side of (3.16) and in estimates (3.18), (3.19), and (3.21) we estimate the integrals with $x_{N}^{2}(\nabla \Delta u)^{2}$ and with $x_{N}^{2}(\Delta u)^{2}$ from relations (3.15) and (3.13) correspondingly. We obtain

$$
\begin{gathered}
\int_{Q_{q R}}\left(\nabla u_{t}\right)^{2} \eta^{s} d x d t \leq \varepsilon_{3} \int_{Q_{q^{3} R}} x_{N}^{2}\left(\Delta u_{t}\right)^{2} d x d t+ \\
+\frac{C_{q}}{\varepsilon_{3} R^{2}} \int_{Q_{q^{3} R}} u_{t}^{2} d x d t+\frac{C_{q}}{\varepsilon_{2} \varepsilon_{3} R^{4}} \int_{Q_{q^{3} R}}(\nabla u)^{2} d x d t+\frac{C_{q}}{R^{6} \varepsilon_{3}} \int_{Q_{q^{3} R}} u^{2} d x d t .
\end{gathered}
$$

Or, in view of the properties of $\eta$ and as $q$ is arbitrary, finally

$$
\begin{gathered}
\int_{Q_{R}}\left(\nabla u_{t}\right)^{2} \eta^{s} d x d t \leq \varepsilon_{3} \int_{Q_{q R}} x_{N}^{2}\left(\Delta u_{t}\right)^{2} d x d t+ \\
+\frac{C_{q}}{\varepsilon_{3} R^{2}} \int_{Q_{q R}} u_{t}^{2} d x d t+\frac{C_{q}}{\varepsilon_{2} \varepsilon_{3} R^{4}} \int_{Q_{q R}}(\nabla u)^{2} d x d t+\frac{C_{q}}{R^{6} \varepsilon_{3}} \int_{Q_{q R}} u^{2} d x d t .
\end{gathered}
$$

Let us proceed. Let $\eta$ be again the same function as before. Multiply equation by (1.1) by $u_{t} \eta^{s}$ and integrate by parts with respect to the $x$-variables:

$$
\begin{gathered}
\int_{Q_{q R}} u_{t}^{2} \eta^{s} d x d t-\int_{Q_{q R}} x_{N}^{2} \nabla \Delta u \nabla u_{t} \eta^{s} d x d t-s \int_{Q_{q R}} x_{N}^{2} \nabla \Delta u \nabla \eta u_{t} \eta^{s-1} d x d t+ \\
+\beta \int_{Q_{q R}} \nabla u \nabla u_{t} \eta^{s} d x d t+\beta s \int_{Q_{q R}} \nabla u \nabla \eta u_{t} \eta^{s-1} d x d t=0 .
\end{gathered}
$$

Integrating once again by parts in the second term with respect to the $x$-variables, we can represent this equality in the form

$$
\int_{Q_{q R}} u_{t}^{2} \eta^{s} d x d t+\int_{Q_{q R}} x_{N}^{2} \Delta u \Delta u_{t} \eta^{s} d x d t+\beta \int_{Q_{q R}} \nabla u \nabla u_{t} \eta^{s} d x d t=
$$




$$
\begin{gathered}
=s \int_{Q_{q R}} x_{N}^{2} \nabla \Delta u \nabla \eta u_{t} \eta^{s-1} d x d t-\beta s \int_{Q_{q R}} \nabla u \nabla \eta u_{t} \eta^{s-1} d x d t-2 \int_{Q_{q R}} x_{N} \Delta u \frac{\partial u_{t}}{\partial x_{N}} \eta^{s} d x d t- \\
-s \int_{Q_{q R}} x_{N}^{2} \Delta u \nabla u_{t} \nabla \eta \eta^{s-1} d x d t .
\end{gathered}
$$

Integrating by parts with respect to the $t$-variable in the second and in the third terms on the left and moving the results to the right, we obtain

$$
\begin{gathered}
\int_{Q_{q R}} u_{t}^{2} \eta^{s} d x d t=s \int_{Q_{q R}} x_{N}^{2} \nabla \Delta u \nabla \eta u_{t} \eta^{s-1} d x d t-s \int_{Q_{q R}} x_{N}^{2} \Delta u \nabla u_{t} \nabla \eta \eta^{s-1} d x d t- \\
-2 \int_{Q_{q R}} x_{N} \Delta u \frac{\partial u_{t}}{\partial x_{N}} \eta^{s} d x d t+\frac{1}{2} \int_{Q_{q R}} x_{N}^{2}(\Delta u)^{2} \eta_{t} \eta^{s-1} d x d t-\beta s \int_{Q_{q R}} \nabla u \nabla \eta u_{t} \eta^{s-1} d x d t+ \\
+\frac{\beta}{2} \int_{Q_{q R}}(\nabla u)^{2} \eta_{t} \eta^{s-1} d x d t \equiv I_{1}+I_{2}+I_{3}+I_{4}+I_{5}+I_{6} .
\end{gathered}
$$

We estimate the integrals $I_{1}-I_{6}$ in the same way as before. We have:

$$
\left|I_{1}\right| \leq \varepsilon \int_{Q_{q R}} u_{t}^{2} \eta^{s} d x d t+\frac{C_{q}}{\varepsilon} \int_{Q_{q R}} x_{N}^{2}(\nabla \Delta u)^{2} d x d t
$$

where we took into account that $x_{N} \leq R$ on $Q_{q R}$. Choosing, for example, $\varepsilon=\frac{1}{10}$ and estimating the second integral from inequality (3.15), we obtain

$$
\left|I_{1}\right| \leq \frac{1}{10} \int_{Q_{q R}} u_{t}^{2} \eta^{s} d x d t+\varepsilon_{2} \int_{Q_{q^{2} R}} u_{t}^{2} d x d t+\frac{C_{q}}{\varepsilon_{2} R^{2}} \int_{Q_{q^{2} R}}(\nabla u)^{2} d x d t+\frac{C_{q}}{R^{4}} \int_{Q_{q^{2} R}} u^{2} d x d t .
$$

Further, for $I_{2}$, taking again into account that $x_{N} \leq R$ on $Q_{q R}$, we have

$$
\left|I_{2}\right| \leq \theta \int_{Q_{q R}}\left(\nabla u_{t}\right)^{2} d x d t+\frac{C_{q}}{\theta} \int_{Q_{q R}} x_{N}^{2}(\Delta u)^{2} d x d t .
$$

Choosing here $\theta=\varepsilon_{4} R^{2}$ and using (3.22), (3.13), we obtain

$$
\left|I_{2}\right| \leq \frac{\varepsilon_{4} C_{q}}{\varepsilon_{3}} \int_{Q_{q^{2} R}} u_{t}^{2} d x d t+\varepsilon_{3} \varepsilon_{4} R^{2} \int_{Q_{q^{2} R}} x_{N}^{2}\left(\Delta u_{t}\right)^{2} d x d t+
$$




$$
+\left(\frac{\varepsilon_{4}}{\varepsilon_{2} \varepsilon_{3}}+\frac{1}{\varepsilon_{4}}\right) \frac{C_{q}}{R^{2}} \int_{Q_{q^{2} R}}(\nabla u)^{2} d x d t+\left(\frac{\varepsilon_{4}}{\varepsilon_{3}}+\frac{1}{\varepsilon_{4}}\right) \frac{C_{q}}{R^{4}} \int_{Q_{q^{2} R}} u^{2} d x d t,
$$

or, choosing $\varepsilon_{4}=\varepsilon_{5} \frac{\varepsilon_{3}}{C_{q}}$

$$
\begin{gathered}
\left|I_{2}\right| \leq \varepsilon_{5} \int_{Q_{q^{2} R}} u_{t}^{2} d x d t+\varepsilon_{5} \varepsilon_{3}^{2} C_{q} R^{2} \int_{Q_{q^{2} R}} x_{N}^{2}\left(\Delta u_{t}\right)^{2} d x d t+ \\
+\left(\frac{\varepsilon_{5}}{\varepsilon_{2}}+\frac{1}{\varepsilon_{5} \varepsilon_{3}}\right) \frac{C_{q}}{R^{2}} \int_{Q_{q^{2} R}}(\nabla u)^{2} d x d t+\left(\varepsilon_{5}+\frac{1}{\varepsilon_{5} \varepsilon_{3}}\right) \frac{C_{q}}{R^{4}} \int_{Q_{q^{2} R}} u^{2} d x d t .
\end{gathered}
$$

The integral $I_{3}$ is estimated completely analogously to $I_{2}$, which gives

$$
\begin{gathered}
\left|I_{3}\right| \leq \varepsilon_{5} \int_{Q_{q^{2} R}} u_{t}^{2} d x d t+\varepsilon_{5} \varepsilon_{3}^{2} C_{q} R^{2} \int_{Q_{q^{2} R}} x_{N}^{2}\left(\Delta u_{t}\right)^{2} d x d t+ \\
+\left(\frac{\varepsilon_{5}}{\varepsilon_{2}}+\frac{1}{\varepsilon_{5} \varepsilon_{3}}\right) \frac{C_{q}}{R^{2}} \int_{Q_{q^{2} R}}(\nabla u)^{2} d x d t+\left(\varepsilon_{5}+\frac{1}{\varepsilon_{5} \varepsilon_{3}}\right) \frac{C_{q}}{R^{4}} \int_{Q_{q^{2} R}} u^{2} d x d t .
\end{gathered}
$$

For $I_{4}$, using again (3.13), we have

$$
\begin{gathered}
\left|I_{4}\right| \leq \frac{C_{q}}{R^{2}} \int_{Q_{q R}} x_{N}^{2}(\Delta u)^{2} d x d t \leq \\
\leq \frac{C_{q}}{R^{2}} \int_{Q_{q^{2} R}}(\nabla u)^{2} d x d t+\frac{C_{q}}{R^{4}} \int_{Q_{q^{2} R}} u^{2} d x d t .
\end{gathered}
$$

Further,

$$
\begin{gathered}
\left|I_{5}\right| \leq \frac{1}{10} \int_{Q_{q R}} u_{t}^{2} \eta^{s} d x d t+\frac{C_{q}}{R^{2}} \int_{Q_{q R}}(\nabla u)^{2} d x d t \\
\left|I_{6}\right| \leq \frac{C_{q}}{R^{2}} \int_{Q_{q R}}(\nabla u)^{2} d x d t .
\end{gathered}
$$

We use relations (3.24) - (3.29) to estimate the right hand side of (3.23) and move the terms with $u_{t}^{2} \eta^{s}$ to the left. As $q$ is arbitrary, taking into account the properties of $\eta$, we obtain 


$$
\begin{gathered}
\int_{Q_{R}} u_{t}^{2} d x d t \leq \varepsilon_{5} \int_{Q_{q R}} u_{t}^{2} d x d t+\varepsilon_{5} \varepsilon_{3}^{2} C_{q} R^{2} \int_{Q_{q R}} x_{N}^{2}\left(\Delta u_{t}\right)^{2} d x d t+ \\
+\left(\frac{\varepsilon_{5}}{\varepsilon_{2}}+\frac{1}{\varepsilon_{5} \varepsilon_{3}}\right) \frac{C_{q}}{R^{2}} \int_{Q_{q^{2} R}}(\nabla u)^{2} d x d t+\left(\varepsilon_{5}+\frac{1}{\varepsilon_{5} \varepsilon_{3}}\right) \frac{C_{q}}{R^{4}} \int_{Q_{q^{2} R}} u^{2} d x d t .
\end{gathered}
$$

Choosing here $\varepsilon_{5} \lessdot 1$ and using Lemma 6, we arrive at the estimate

$$
\begin{gathered}
\int_{Q_{R}} u_{t}^{2} d x d t \leq \varepsilon_{3}^{2} C_{q} R^{2} \int_{Q_{q R}} x_{N}^{2}\left(\Delta u_{t}\right)^{2} d x d t+ \\
+\frac{1}{\varepsilon_{2} \varepsilon_{3}} \frac{C_{q}}{R^{2}} \int_{Q_{q R}}(\nabla u)^{2} d x d t+\left(1+\frac{1}{\varepsilon_{3}}\right) \frac{C_{q}}{R^{4}} \int_{Q_{q R}} u^{2} d x d t .
\end{gathered}
$$

To obtain one more integral inequality multiply equation (1.1) by $\left[\nabla\left(x_{N}^{2} \nabla \Delta u\right)_{t}\right] \eta^{s}$ with subsequent integration and represent the result as

$$
\begin{gathered}
\int_{Q_{R}} u_{t} \nabla\left(x_{N}^{2} \nabla \Delta u_{t}\right) \eta^{s} d x d t+\frac{1}{2} \int_{Q_{R}}\left(\left[\nabla\left(x_{N}^{2} \nabla \Delta u\right)\right]^{2}\right)_{t} \eta^{s} d x d t- \\
-\beta \int_{Q_{R}} \nabla\left(x_{N}^{2} \nabla \Delta u\right)_{t} \Delta u \eta^{s} d x d t=0 .
\end{gathered}
$$

Integrating by parts with respect to the $x$-variables in the first and in the third integrals and integrating by parts with respect to the $t$-variable in the second integral, we obtain

$$
\begin{gathered}
-\int_{Q_{q R}} x_{N}^{2} \nabla u_{t} \nabla \Delta u_{t} \eta^{s} d x d t+\frac{\beta}{2} \int_{Q_{q R}} x_{N}^{2}(\nabla \Delta u)_{t}^{2} \eta^{s} d x d t= \\
=s \int_{Q_{q R}} x_{N}^{2} u_{t} \nabla \Delta u_{t} \nabla \eta \eta^{s-1} d x d t+\frac{s}{2} \int_{Q_{q R}}\left[\nabla\left(x_{N}^{2} \nabla \Delta u\right)\right]^{2} \eta_{t} \eta^{s-1} d x d t- \\
-\beta s \int_{Q_{q R}} x_{N}^{2} \nabla \Delta u_{t} \nabla \eta \eta^{s-1} \Delta u d x d t .
\end{gathered}
$$

We transform the resulting equation as follows. First we integrate by parts with respect to the $x$-variables in the first term on the left and in the first term on the right. The integral over the surface $\left\{x_{N}=0\right\}$ vanishes in view of condition 
(1.2) and we recall that this condition is valid under assumptions of the lemma for the function $u$ and for it's derivative $u_{t}$. Besides, we integrate by parts with respect to the $t$-variable in the second term on the left and in the last term on the right. Finally, using equation (1.1), we just replace in the second term on the right $\nabla\left(x_{N}^{2} \nabla \Delta u\right)=-u_{t}+\beta \Delta u$. As the result we get the equality

$$
\begin{aligned}
& \int_{Q_{q R}} x_{N}^{2}\left(\Delta u_{t}\right)^{2} \eta^{s} d x d t=-2 \int_{Q_{q R}} x_{N}\left(u_{t}\right)_{x_{N}} \Delta u_{t} \eta^{s} d x d t-s \int_{Q_{q R}} x_{N}^{2} \nabla u_{t} \Delta u_{t} \nabla \eta \eta^{s-1} d x d t+ \\
& +\frac{\beta s}{2} \int_{Q_{q R}} x_{N}^{2}(\nabla \Delta u)^{2} \eta_{t} \eta^{s-1} d x d t-2 s \int_{Q_{q R}} x_{N} u_{t} \Delta u_{t} \eta_{x_{N}} \eta^{s-1} d x d t-s \int_{Q_{q R}} x_{N}^{2} \nabla u_{t} \Delta u_{t} \nabla \eta \eta^{s-1} d x d t- \\
& \quad-s \int_{Q_{q R}} x_{N}^{2} u_{t} \Delta u_{t} \nabla\left(\nabla \eta \eta^{s-1}\right) d x d t+\frac{s}{2} \int_{Q_{q R}}\left[-u_{t}+\beta \Delta u\right]^{2} \eta_{t} \eta^{s-1} d x d t+ \\
& +\beta s \int_{Q_{q R}} x_{N}^{2} \nabla \Delta u\left(\nabla \eta \eta^{s-1}\right)_{t} \Delta u d x d t+\beta s \int_{Q_{q R}} x_{N}^{2} \nabla \Delta u \nabla \eta \eta^{s-1} \Delta u_{t} d x d t \equiv \sum_{k=1}^{9} I_{k} .
\end{aligned}
$$

The integrals $I_{k}$ are estimated according to the same schema as above. We have

$$
\begin{gathered}
\left|I_{1}\right|+\left|I_{2}\right|+\left|I_{5}\right| \leq \varepsilon \int_{Q_{q R}} x_{N}^{2}\left(\Delta u_{t}\right)^{2} \eta^{s} d x d t+\frac{C}{\varepsilon} \int_{Q_{q R}}\left(\nabla u_{t}\right)^{2} d x d t \leq \\
\quad \leq \varepsilon \int_{Q_{q R}} x_{N}^{2}\left(\Delta u_{t}\right)^{2} \eta^{s} d x d t+\varepsilon_{3} \frac{C_{q}}{\varepsilon} \int_{Q_{q^{2} R}} x_{N}^{2}\left(\Delta u_{t}\right)^{2} d x d t+ \\
+\frac{C_{q}}{\varepsilon \varepsilon_{3} R^{2}} \int_{Q_{q^{2} R}} u_{t}^{2} d x d t+\frac{C_{q}}{\varepsilon \varepsilon_{2} \varepsilon_{3} R^{4}} \int_{Q_{q^{2} R}}(\nabla u)^{2} d x d t+\frac{C_{q}}{\varepsilon R^{6} \varepsilon_{3}} \int_{Q_{q^{2} R}} u^{2} d x d t,
\end{gathered}
$$

where we made use of (3.22). Further,

$$
\left|I_{3}\right| \leq \frac{C_{q}}{R^{2}} \int_{Q_{q R}} x_{N}^{2}(\nabla \Delta u)^{2} d x d t \leq
$$




$$
\leq \frac{\varepsilon_{2} C_{q}}{R^{2}} \int_{Q_{q^{2} R}} u_{t}^{2} d x d t+\frac{C_{q}}{\varepsilon_{2} R^{4}} \int_{Q_{q^{2} R}}(\nabla u)^{2} d x d t+\frac{C_{q}}{R^{6}} \int_{Q_{q^{2} R}} u^{2} d x d t
$$

where we took into account estimate (3.15). Further,

$$
\begin{array}{r}
\left|I_{4}\right|+\left|I_{6}\right| \leq \varepsilon \int_{Q_{q R}} x_{N}^{2}\left(\Delta u_{t}\right)^{2} \eta^{s} d x d t+\frac{C_{q}}{\varepsilon R^{2}} \int_{Q_{q R}} u_{t}^{2} d x d t \\
\left|I_{7}\right| \leq \frac{C_{q}}{R^{2}} \int_{Q_{q R}} u_{t}^{2} d x d t+\beta \frac{C_{q}}{R^{2}}\left(\beta \int_{Q_{q R}}(\Delta u)^{2} d x d t\right) \leq \\
\leq \frac{C_{q}}{R^{2}} \int_{Q_{q^{2} R}} u_{t}^{2} d x d t+\frac{C_{q}}{\varepsilon_{2} R^{4}} \int_{Q_{q^{2} R}}(\nabla u)^{2} d x d t+\frac{C_{q}}{R^{6}} \int_{Q_{q^{2} R}} u^{2} d x d t,
\end{array}
$$

where we made use of (3.15). For the next integral we have

$$
\begin{array}{r}
\left|I_{8}\right| \leq \frac{C_{q}}{R^{2}}\left(\int_{Q_{q R}} x_{N}^{2}(\nabla \Delta u)^{2} d x d t+\beta \int_{Q_{q R}}(\Delta u)^{2} d x d t\right) \leq \\
\leq \frac{\varepsilon_{2} C_{q}}{R^{2}} \int_{Q_{q^{2} R}} u_{t}^{2} d x d t+\frac{C_{q}}{\varepsilon_{2} R^{4}} \int_{Q_{q^{2} R}}(\nabla u)^{2} d x d t+\frac{C_{q}}{R^{6}} \int_{Q_{q^{2} R}} u^{2} d x d t,
\end{array}
$$

which is analogous to (3.15). Further, as before

$$
\begin{gathered}
\left|I_{9}\right| \leq \varepsilon \int_{Q_{q R}} x_{N}^{2}\left(\Delta u_{t}\right)^{2} \eta^{s} d x d t+\frac{C_{q}}{\varepsilon R^{2}} \int_{Q_{q R}} x_{N}^{2}(\nabla \Delta u)^{2} d x d t \leq \\
\leq \varepsilon \int_{Q_{q R}} x_{N}^{2}\left(\Delta u_{t}\right)^{2} \eta^{s} d x d t+ \\
+\frac{\varepsilon_{2} C_{q}}{\varepsilon R^{2}} \int_{Q_{q^{2} R}} u_{t}^{2} d x d t+\frac{C_{q}}{\varepsilon_{2} \varepsilon R^{4}} \int_{Q_{q^{2} R}}(\nabla u)^{2} d x d t+\frac{C_{q}}{\varepsilon R^{6}} \int_{Q_{q^{2} R}} u^{2} d x d t .
\end{gathered}
$$

The estimate for the left hand side of (3.31) follows from estimates (3.32)(3.37). We substitute these estimates in (3.31), then we choose sufficiently 
small $\varepsilon$ in (3.32), (3.34), and (3.37) and move the corresponding integrals with $x_{N}^{2}\left(\Delta u_{t}\right)^{2} \eta^{s}$ to the left hand side of (3.31). We also estimate the integrals over $Q_{q R}$ on the right hand side of (3.31) by the same integrals over $Q_{q^{2} R}$ and we denote $q^{2}$ again by $q$ (as $q$ is arbitrary). As a result, we obtain the estimate

$$
\begin{gathered}
\int_{Q_{R}} x_{N}^{2}\left(\Delta u_{t}\right)^{2} d x d t \leq \varepsilon_{3} C_{q} \int_{Q_{q R}} x_{N}^{2}\left(\Delta u_{t}\right)^{2} d x d t+ \\
+\frac{\left(1+\varepsilon_{2}\right) C_{q}}{\varepsilon_{3} R^{2}} \int_{Q_{q R}} u_{t}^{2} d x d t+\frac{C_{q}}{\varepsilon_{3} \varepsilon_{2} R^{4}} \int_{Q_{q R}}(\nabla u)^{2} d x d t+\frac{C_{q}}{\varepsilon_{3} R^{6}} \int_{Q_{q R}} u^{2} d x d t .
\end{gathered}
$$

Choosing here and in (3.30) $\varepsilon_{3}=\theta C_{q}^{-1}, \theta \in(0,1 / 2)$ and making use of Lemma 6. we arrive at the estimate

$$
\begin{gathered}
\int_{Q_{R}} x_{N}^{2}\left(\Delta u_{t}\right)^{2} d x d t \leq \\
\leq \frac{\left(1+\varepsilon_{2}\right) C_{q}}{R^{2} \theta} \int_{Q_{q R}} u_{t}^{2} d x d t+\frac{C_{q}}{\varepsilon_{2} R^{4} \theta} \int_{Q_{q R}}(\nabla u)^{2} d x d t+\frac{C_{q}}{R^{6} \theta} \int_{Q_{q R}} u^{2} d x d t .
\end{gathered}
$$

Now we will combine our estimates to prove (2.1). Under our choice of $\varepsilon_{3}=$ $\theta C_{q}^{-1}$ it follows from (3.30) and (3.38) that for $\varepsilon_{2}<1$

$$
\begin{aligned}
\int_{Q_{R}} u_{t}^{2} d x d t \leq & \frac{\theta^{2}}{C_{q}} R^{2}\left(\frac{\left(1+\varepsilon_{2}\right) C_{q}}{R^{2} \theta} \int_{Q_{q^{2} R}} u_{t}^{2} d x d t+\frac{C_{q} C}{\varepsilon_{2} R^{4} \theta} \int_{Q_{q^{2} R}}(\nabla u)^{2} d x d t+\frac{C_{q}}{R^{6} \theta} \int_{Q_{q R}} u^{2} d x d t\right)+ \\
& +\frac{1}{\varepsilon_{2} \theta} \frac{C_{q}}{R^{2}} \int_{Q_{q R}}(\nabla u)^{2} d x d t+\left(1+\frac{1}{\theta}\right) \frac{C_{q}}{R^{4}} \int_{Q_{q R}} u^{2} d x d t \leq \\
\leq & \theta \int_{Q_{q^{2} R}} u_{t}^{2} d x d t+\frac{C_{q}}{\varepsilon_{2} \theta R^{2}} \int_{Q_{q^{2} R}}(\nabla u)^{2} d x d t+\frac{C_{q}}{\theta R^{4}} \int_{Q_{q^{2} R}} u^{2} d x d t .
\end{aligned}
$$

Denoting here $q^{2}$ again by $q$ and applying Lemma 6, we get the estimate

$$
\int_{Q_{R}} u_{t}^{2} d x d t \leq \frac{C_{q}}{\varepsilon_{2} R^{2}} \int_{Q_{q R}}(\nabla u)^{2} d x d t+\frac{C_{q}}{R^{4}} \int_{Q_{q R}} u^{2} d x d t .
$$

Substitute now estimates (3.13) an (3.15) in estimate (3.9) and take into account (3.15). We obtain 


$$
\begin{aligned}
& \int_{Q_{R}}|\nabla u|^{2} d x d t \leq \\
& \leq \varepsilon_{1} R^{2}\left(\varepsilon_{2} \int_{Q_{q^{2} R}} u_{t}^{2} d x d t+\frac{C_{q}}{\varepsilon_{2} R^{2}} \int_{Q_{q^{2} R}}(\nabla u)^{2} d x d t+\frac{C_{q}}{R^{4}} \int_{Q_{q^{2} R}} u^{2} d x d t\right)+ \\
& +\varepsilon_{1}\left(C_{q} \int_{Q_{q^{2} R}}|\nabla u|^{2} d x d t+\frac{C_{q}}{R^{2}} \int_{Q_{q^{2} R}} u^{2} d x d t\right)+\frac{C_{q}}{\varepsilon_{1} R^{2}} \int_{Q_{q R}} u^{2} d x d t \leq \\
& \leq \varepsilon_{1} R^{2} \varepsilon_{2}\left(\frac{C_{q}}{\varepsilon_{2} R^{2}} \int_{Q_{q^{3} R}}(\nabla u)^{2} d x d t+\frac{C_{q}}{R^{4}} \int_{Q_{q^{3} R}} u^{2} d x d t\right)+ \\
& +\frac{\varepsilon_{1} C_{q}}{\varepsilon_{2}} \int_{Q_{q^{2} R}}(\nabla u)^{2} d x d t+\frac{C_{q}}{\varepsilon_{1} R^{2}} \int_{Q_{q^{2} R}} u^{2} d x d t \leq \\
& \leq \frac{\varepsilon_{1} C_{q}}{\varepsilon_{2}} \int_{Q_{q^{3} R}}(\nabla u)^{2} d x d t+\frac{C_{q}}{\varepsilon_{1} R^{2}} \int_{Q_{q^{3} R}} u^{2} d x d t .
\end{aligned}
$$

Denoting here $q^{3}$ again by $q$, choosing $\varepsilon_{1}=\varepsilon \varepsilon_{2} C_{q}^{-1}$, an applying Lemma [6, we obtain finally

$$
\int_{Q_{R}}|\nabla u|^{2} d x d t \leq \frac{C_{q}}{R^{2}} \int_{Q_{q R}} u^{2} d x d t,
$$

that is exactly the first from inequalities (2.1). The second from these inequalities follows now from (3.39).

Thus, Lemma 4 is proved and this finishes also the proof of Theorem 1.

\section{References}

[1] D.E.Edmunds, L.A.Peletier. A Liouville theorem for degenerate elliptic equations// J. London Math. Soc. (2) 7 (1973), 95-100.

[2] Tianling Jin, Jingang Xiong. A Liouville Theorem for Solutions of Degenerate Monge-Ampe're Equations// Comm. Partial Differential Equations 39 (2014), no. 2, 306-320. 
[3] Genggeng Huang. A Liouville theorem of degenerate elliptic equation and its application// Discrete Contin. Dyn. Syst. 33 (2013), no. 10, 4549-4566.

[4] G.DHAZ. A note on the Liouville method applied to elliptic eventually degenerate fully nonlinear equations governed by the Pucci operators and the Keller-Osserman condition// Math. Ann. 353 (2012), no. 1, 145-159.

[5] S. D.Eidelman, A.P.Malickaja. Liouville theorems for a certain class of degenerate parabolic equations (Russian)// Mathematics collection (Russian), pp. 250-253. Izdat. "Naukova Dumka", Kiev, 1976.

[6] Shapoval, A.B. Liouville's theorem for a second-order elliptic equation with degenerate coefficients// Vestnik Moskov. Univ. Ser. I Mat. Mekh. 1998, no. 2, 21-26; translation in Moscow Univ. Math. Bull. 53 (1998), no. 2, 22-27.

[7] E.DiBenedetto, U.Gianazza, V.Vespri. Liouville-type theorems for certain degenerate and singular parabolic equations// C. R. Math. Acad. Sci. Paris 348 (2010), no. 15-16, 873-877.

[8] L.Moschini. New Liouville theorems for linear second order degenerate elliptic equations in divergence form// Ann. Inst. H. Poincare' Anal. Non Line'aire 22 (2005), no. 1, 11-23.

[9] Yu.T. Kuz'menko. The Liouville theorem for degenerate elliptic and parabolic equations (Russian)// Mat. Zametki 29 (1981), no. 3, 397-408,

[10] I.M. Kolodii. The Liouville theorem for generalized solutions of degenerate uasilinear parabolic equations. (Russian)// Differentsial'nye Uravneniya 21 (1985), no. 5, 841-854.

[11] H. Knüpfer. Well-posedness for the Navier slip thin-film equation in the case of partial wetting// Comm. Pure Appl. Math. 64 (2011), no. 9, 1263-1296.

[12] M. Boutat, S. Hilout, J.-E. Rakotoson, J.-M. Rakotoson. A generalized thinfilm equation in multidimensional space// Nonlinear Anal., 69 (2008), no. 4, 1268-1286.

[13] M. Bertsch, L, Giacomelli, G. Karali. Thin-film equations with "partial wetting" energy: existence of weak solutions// Phys. D, 209 (2005), no. 1-4, $17-27$.

[14] R. Dal Passo, H. Garcke, G. Grün. On a fourth-order degenerate parabolic equation: global entropy estimates, existence, and qualitative behavior of solutions// SIAM J. Math. Anal., 29 (1998), no. 2, 321-342.

[15] B. Liang. Mathematical analysis to a nonlinear fourth-order partial differential equation// Nonlinear Anal., 74 (2011), no. 11, 3815-3828. 
[16] M.Giaquinta. Multiple integrals in the calculus of variations and nonlinear elliptic systems// Annals of Mathematics Studies, 105. Princeton University Press, Princeton, NJ, 1983. vii +297 pp.

[17] D. Andreucci, A. Tedeev. Finite speed of propagation for the thin-film equation and other higher-order parabolic equations with general nonlinearity// Interfaces Free Bound., 3 (2001), no. 3, 233-264.

[18] D. Andreucci, A. Tedeev. Universal bounds at the blow-up time for nonlinear parabolic equations// Adv. Differential Equations, 10 (2005), no. 1, 89-120.

[19] S.P. Degtyarev, A.F. Tedeev. $L_{1}--L_{\infty}$ estimates for the solution of the Cauchy problem for an anisotropic degenerate parabolic equation with double nonlinearity and growing initial data// Sb. Math., 198 (2007), no. 5-6, 639-660.

[20] S.P. Degtyarev, A.F. Tedeev. Two-sided estimates for the support of a solution of the Cauchy problem for an anisotropic quasilinear degenerate equation// Ukrainian Math. J., 58 (2006), no. 11, 1673-1684.

[21] S.P. Degtyarev, A.F. Tedeev. Estimates for the solution of the Cauchy problem with increasing initial data for a parabolic equation with anisotropic degeneration and double nonlinearity// Dokl. Math., 76 (2007), no. 3, 824827.

[22] S.P. Degtyarev. On conditions for the instantaneous compactification of the support of the solution and on sharp estimates for the support in the Cauchy problem for a parabolic equation with double nonlinearity and absorption. Sb. Math., 199 (2008), no. 3-4, 511-538.

[23] S.P. Degtyarev. Instantaneous support shrinking phenomenon in the case of fast diffusion for a doubly nonlinear parabolic equation with absorption// Adv. Differential Equations, 13 (2008), no. 11-12, 1031-1050.

[24] S.P. Degtyarev. On the instantaneous shrinking of the support of a solution in the Cauchy problem for an anisotropic parabolic equation// Ukrainian Math. J., 61 (2009), no. 5, 747-763.

[25] S.P. Degtyarev. The effect of nonhomogeneous absorption on the instantaneous shrinking of the support in the Cauchy problem for a quasilinear degenerate equation// Ukr. Math. Bull., 6 (2009), no. 3, 335-366.

[26] V.G. Maz'ja. Sobolev spaces// Springer Series in Soviet Mathematics., Springer-Verlag, Berlin, 1985. xix +486 pp. 
[27] R. Adams, John J. F. Fournier. Sobolev spaces// Second edition. Pure and Applied Mathematics (Amsterdam), 140. Elsevier/Academic Press, Amsterdam, 2003. $x i v+305 \mathrm{pp}$.

[28] A. Kufner, L.-E. Persson. Weighted inequalities of Hardy type// World Scientific Publishing Co., Inc., River Edge, NJ, 2003. xviii+357 pp. 